\title{
Investigation of Combi Product Fungicides for the Management of Blast Diseases of Paddy
}

\author{
G.N. Hosagoudar* \\ Junior Rice Pathologist, AICRIP on Rice, Agricultural and Horticultural Research Station, \\ Ponnampet, Kodagu-571216, Karnataka, India \\ *Corresponding author
}

A B S T R A C T

\section{Keywords \\ Management, Blast, Disease, Combi product and Fungicides \\ Article Info \\ Accepted: \\ 17 June 2019 \\ Available Online: \\ 10 July 2019}

\begin{abstract}
Rice (Oryza sativa L.) is an important cereal crop belonging to the grass family Poaceae. An experiment was conducted during Kharif 2017 and 2018 to know the impact of nine fungicide treatments. Among nine treatments, Azoxystrobin $18.2 \% \mathrm{w} / \mathrm{w}+$ Difenoconazole $11.4 \%$ w/w SC @ $1.0 \mathrm{~g} / \mathrm{L}$ and Trifloxystrobin 25\% + Tebuconazole 50\% WG @ $0.4 \mathrm{~g} / \mathrm{L}$ were on par with each other with least pooled leaf blast disease index of $20.03 \%$ and $20.90 \%$ and neck blast disease incidence of $16.48 \%$ and $16.93 \%$ respectively followed by Tricyclazole 75\% WP @ $0.6 \mathrm{~g} / \mathrm{L}$. Further, the highest pooled yield was recorded in Trifloxystrobin 25\% + Tebuconazole 50\% WG @ $0.4 \mathrm{~g} / \mathrm{L}(4311.85 \mathrm{~kg} / \mathrm{ha})$ followed by Azoxystrobin 18.2\% w/w + Difenoconazole 11.4\% w/w SC @ 1.0 g/L (4310.74 kg/ ha). However, when cost benefit ratio was calculated, Trifloxystrobin $25 \%+$ Tebuconazole 50\% WG (1.58) and Azoxystrobin $18.2 \% \mathrm{w} / \mathrm{w}+$ Difenoconazole 11.4\% w/w SC(1.58) respectively followed by Tricyclazole $75 \% \mathrm{WP}(1.46)$.
\end{abstract}

\section{Introduction}

Rice (Oryza sativa L.) is the principal staple food for more than two billion people; most of them live in rural and urban areas of tropical and subtropical Asia. Rice is grown on millions of small farms with an average size ranging from $0.4-3.5$ ha, primarily to meet family needs. Rice is the important cereal crop grown throughout the world and is the second most staple food crop of the world next to wheat and staple food for two third of world's population (1) (Abodolereza and racionzer, 2009).
Starting in 2500 B.C. rice has been a source of food for people. Rice production originated in China, and was spread to countries such as Sri Lanka and India. It is believed that rice was brought to West Asia and Greece in 300 B.C. by Alexander the Great's armies.

China and India account for roughly 50 per cent of the world's total rice area and jointly produce 55 per cent of world's rice. Other major rice-growing countries are Indonesia, Bangladesh, Vietnam, and Thailand, which produce respectively nine, six, five, and four percent of world's rice. 
Projection of India rice production target for $2025 \mathrm{AD}$ is 140 million tons, which can be achieved only by increasing the rice production by over 2 million tons per year in the coming decade and this has to be achieved against back drop of diminishing natural resource such as land and water.

Globally, rice is cultivated with an area about 161.4 million hectare, production of about 633.3 million tonnes with a productivity of 3.14 tonnes per hectare (2).In India area under rice cultivation is 44 million hectare and production of about 104 million tonnes with productivity of about 2.4 tonnes per hectare (2). In Karnataka, rice is cultivated with an area of 13.43 lakh ha, production of 39.53 lakh tonnes and productivity of 3.09 tonnes per hectare (2).

The productivity of rice is highly affected by several biotic and abiotic factors. Rice crop is susceptible to many fungal, bacterial, viral and nematode diseases (3). The most significant disease in rice is blast disease incited by Pyricularia oryzae as it is reported in more than 85 countries wherever rice is grown (4). Heavy yield losses have been reported in many rice growing countries viz., 75,50 and 40 percent grain loss was occur in India (5), Philippines (6) and Nigeria (7). The pathogen can cause damage up to $90 \%$ and sometime total crop loss under favourable conditions (8).

The rice blast fungus can causes symptoms like leaf blast, nodal blast and neck or panicle blast. The most severe stage is neck blast (9). The usual practices followed for management of blast disease of rice includes use of resistant varieties, use of fungicides, application of fertilizers and irrigations (10, 11). Thus, the study was conducted for the management of blast disease of rice under field condition by using new combi product and systemic fungicides.

\section{Materials and Methods}

An experiment was conducted during Kharif 2017 and 2018 at AHRS, Ponnampet. The susceptible variety Intan were sown on $11 / 07 / 2017,16 / 07 / 2018$ and transplanted on $17 / 08 / 2017,19 / 08 / 2018$ respectively in RCBD with 4 replications and 10 treatments. The spacing followed was $15 \times 15 \mathrm{~cm}$ and total plot size were10.12 $\mathrm{m}^{2}$ (Table 1 and Plate 1). Totally two sprays were given, first at appearance of the leaf blast disease as prophalytic spray and second spray at 50\% emergence of the panicles. Five hills were randomly selected from each plot and were tagged. The observations for leaf blast was recorded as PDI after first spray by using 0-9 scale given by IRRI (1996) and for the neck blast as percent neck blast incidence at second spray and at harvest, The leaf blast incidence was calculated by using formula given by (12).

$\mathrm{PDI}=$

Sum of individual rating

Number of
leaves assessed $\quad \begin{gathered}\text { Maximum disease } \\ \text { grade value }\end{gathered}$

From the selected five hills randomly from each plot, the neck blast incident was calculated by using the formula given below.

Per cent neck blast incidence $=$ Infected panicles

Total number of panicles

Statistical analysis was carried out as per the procedure given by (13). The original means were converted into arc sine transformed values. The yield was recorded at harvest in all the treatments. 


\section{Results and Discussion}

The pooled data results obtained indicates that, all the treatments recorded significantly reduced the pooled per cent leaf blast disease index and per cent neck blast disease incidence compared to untreated control. Azoxystrobin 18.2\% w/w +Difenoconazole 11.4\% w/w SC @ $1.0 \mathrm{~g} / \mathrm{L}$ and Trifloxystrobin 25\% + Tebuconazole 50\% WG @ $0.4 \mathrm{~g} / \mathrm{L}$ were on par with each other with least pooled leaf blast disease index of $20.03 \%$ and $20.90 \%$ and neck blast disease incidence of $16.48 \%$ and $16.93 \%$ respectively followed by followed by Tricyclazole 75\% WP @ 0.6 $\mathrm{g} /$ Ltreatment was with pooled leaf blast disease index of $23.25 \%$ and pooled neck blast incidence of $19.65 \%$ when compared to control (61.94\% and $54.95 \%)$.

The maximum leaf blast per cent disease reduction over control (PDC) was observed in Azoxystrobin $18.2 \%$ w/w +Difenoconazole 11.4\% w/w SC @ 1.0 g/L (67.66 PDC) and Trifloxystrobin 25\% + Tebuconazole 50\% WG @ 0.4 g/L(66.26 PDC) followed byTricyclazole $\quad 75 \% \quad$ WP @ 0.6 $\mathrm{g} / \mathrm{L}(62.46 \mathrm{PDC})$. Similarly, the maximum neck blast per cent disease reduction over control (PDC) was observed in Azoxystrobin $18.2 \% \mathrm{w} / \mathrm{w}+$ Difenoconazole $11.4 \% \mathrm{w} / \mathrm{w} \mathrm{SC}$ @ $1.0 \mathrm{~g} / \mathrm{L}$ (70.01PDC) and Trifloxystrobin 25\% + Tebuconazole 50\% WG @ 0.4 g/L (69.19PDC) followed by Tricyclazole $75 \%$ WP @ 0.6 g/L(64.24PDC) (Fig. 1).

Fig.1 Best treatments observed against blast of paddy
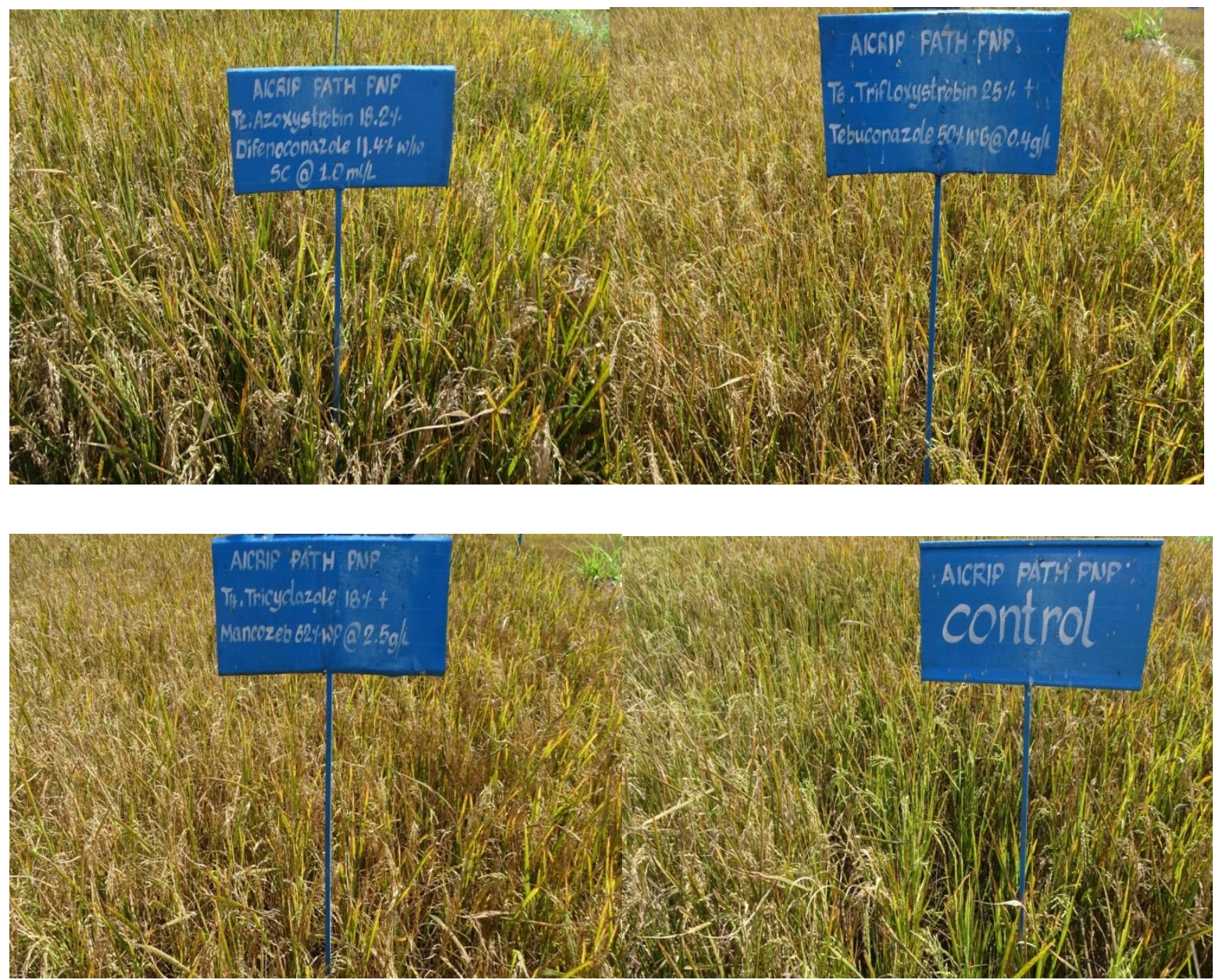
Table.1 Investigation of combi product fungicides for the management of blast diseases of paddy

\begin{tabular}{|c|c|c|c|c|c|c|c|c|c|c|c|c|c|}
\hline \multirow{2}{*}{$\begin{array}{l}\text { Tr. } \\
\text { No. }\end{array}$} & \multirow[t]{2}{*}{ Treatments Details } & \multirow{2}{*}{$\begin{array}{l}\text { Dosage } \\
/ \mathrm{L}\end{array}$} & \multicolumn{3}{|c|}{ Leaf blast PDI } & \multirow{2}{*}{$\begin{array}{l}\text { Leaf } \\
\text { blast } \\
\text { PDC }\end{array}$} & \multicolumn{3}{|c|}{ Neck blast PDI } & \multirow{2}{*}{$\begin{array}{l}\text { Neck } \\
\text { blast } \\
\text { PDC }\end{array}$} & \multicolumn{3}{|c|}{ Grain yield $\mathrm{Kg} / \mathrm{ha}$} \\
\hline & & & 2017 & 2018 & Pooled & & 2017 & 2018 & Pooled & & 2017 & 2018 & Pooled \\
\hline $\mathrm{T}_{1}$ & $\begin{array}{l}\text { Flusilazole } 12.5 \%+ \\
\text { Carbendazim } 25 \% \text { SC }\end{array}$ & $1.0 \mathrm{ml}$ & $\begin{array}{c}26.88 \\
(31.24)^{*}\end{array}$ & $\begin{array}{c}25.68 \\
(30.46)\end{array}$ & $\begin{array}{c}26.28 \\
(30.85)\end{array}$ & 57.57 & $\begin{array}{c}20.28 \\
(26.77)\end{array}$ & $\begin{array}{c}19.28 \\
(26.05)\end{array}$ & $\begin{array}{c}19.78 \\
(26.41)\end{array}$ & 64.00 & 3694.81 & 3843.70 & 3787.78 \\
\hline $\mathrm{T}_{2}$ & $\begin{array}{l}\text { Azoxystrobin } 18.2 \% \mathrm{w} / \mathrm{w} \\
+ \text { Difenoconazole } 11.4 \% \\
\text { w/w SC }\end{array}$ & $1.0 \mathrm{ml}$ & $\begin{array}{c}20.53 \\
(26.87)\end{array}$ & $\begin{array}{c}19.53 \\
(26.14)\end{array}$ & $\begin{array}{c}20.03 \\
(26.51)\end{array}$ & 67.66 & $\begin{array}{c}16.73 \\
(23.97)\end{array}$ & $\begin{array}{c}16.23 \\
(23.54)\end{array}$ & $\begin{array}{c}16.48 \\
(23.76)\end{array}$ & 70.01 & 4211.11 & 4373.33 & 4310.74 \\
\hline $\mathrm{T}_{3}$ & $\begin{array}{l}\text { Azoxystrobin } 11 \%+ \\
\text { Tebuconazole } 18.3 \% \mathrm{w} / \mathrm{w} \\
\text { SC }\end{array}$ & $1.5 \mathrm{ml}$ & $\begin{array}{c}24.63 \\
(29.76)\end{array}$ & $\begin{array}{c}23.80 \\
(29.20)\end{array}$ & $\begin{array}{c}24.21 \\
(29.48)\end{array}$ & 60.91 & $\begin{array}{l}18.90 \\
(25.77)\end{array}$ & $\begin{array}{c}17.83 \\
(24.97)\end{array}$ & $\begin{array}{c}18.36 \\
(25.37)\end{array}$ & 66.59 & 3968.89 & 4117.04 & 4061.48 \\
\hline $\mathrm{T}_{4}$ & $\begin{array}{l}\text { Tricyclazole } 18 \%+ \\
\text { Mancozeb } 62 \% \mathrm{WP}\end{array}$ & $2.5 \mathrm{~g}$ & $\begin{array}{c}31.15 \\
(33.88)\end{array}$ & $\begin{array}{c}29.69 \\
(32.97)\end{array}$ & $\begin{array}{c}30.42 \\
(33.43)\end{array}$ & 50.89 & $\begin{array}{c}26.10 \\
(30.74)\end{array}$ & $\begin{array}{c}25.10 \\
(30.08)\end{array}$ & $\begin{array}{c}25.60 \\
(30.41)\end{array}$ & 53.41 & 3684.44 & 3832.59 & 3777.04 \\
\hline $\mathrm{T}_{5}$ & $\begin{array}{l}\text { Zineb } 68 \%+ \\
\text { Hexaconazole } 4 \% \text { WP }\end{array}$ & $2.5 \mathrm{~g}$ & $\begin{array}{c}34.25 \\
(35.79) \\
\end{array}$ & $\begin{array}{c}32.25 \\
(34.57) \\
\end{array}$ & $\begin{array}{c}33.25 \\
(35.18) \\
\end{array}$ & 46.32 & $\begin{array}{c}24.25 \\
(29.49)\end{array}$ & $\begin{array}{c}23.00 \\
(28.65)\end{array}$ & $\begin{array}{c}23.63 \\
(29.07)\end{array}$ & 57.00 & 3385.19 & 3533.33 & 3477.78 \\
\hline $\mathrm{T}_{6}$ & $\begin{array}{l}\text { Trifloxystrobin } 25 \%+ \\
\text { Tebuconazole } 50 \% \mathrm{WG}\end{array}$ & $0.4 \mathrm{~g}$ & $\begin{array}{c}22.65 \\
(28.35)\end{array}$ & $\begin{array}{c}19.15 \\
(25.85)\end{array}$ & $\begin{array}{c}20.90 \\
(27.12)\end{array}$ & 66.26 & $\begin{array}{c}18.93 \\
(25.64)\end{array}$ & $\begin{array}{c}14.93 \\
(22.58)\end{array}$ & $\begin{array}{c}16.93 \\
(24.15)\end{array}$ & 69.19 & 4034.07 & 4478.52 & 4311.85 \\
\hline $\mathrm{T}_{7}$ & $\begin{array}{l}\text { Mancozeb } 50 \%+ \\
\text { Carbendazium } 25 \% \text { WS }\end{array}$ & $2.5 \mathrm{~g}$ & $\begin{array}{c}33.25 \\
(35.18)\end{array}$ & $\begin{array}{c}31.75 \\
(34.26) \\
\end{array}$ & $\begin{array}{c}32.50 \\
(34.72)\end{array}$ & 47.53 & $\begin{array}{l}23.25 \\
(28.81)\end{array}$ & $\begin{array}{c}22.25 \\
(28.13)\end{array}$ & $\begin{array}{c}22.75 \\
(28.47)\end{array}$ & 58.60 & 3533.33 & 3681.48 & 3625.93 \\
\hline $\mathrm{T}_{8}$ & $\begin{array}{l}\text { Fluxapyroxad } 62.5 \mathrm{~g} / \mathrm{l}+ \\
\text { Epoxiconazole } 62.5 \mathrm{~g} / \mathrm{l} \mathrm{EC}\end{array}$ & $1.5 \mathrm{ml}$ & $\begin{array}{c}28.43 \\
(32.17)\end{array}$ & $\begin{array}{c}27.18 \\
(31.37)\end{array}$ & $\begin{array}{c}27.80 \\
(31.77)\end{array}$ & 55.12 & $\begin{array}{c}23.25 \\
(28.79)\end{array}$ & $\begin{array}{c}22.25 \\
(28.11)\end{array}$ & $\begin{array}{c}22.75 \\
(28.45)\end{array}$ & 58.60 & 3829.63 & 3977.78 & 3922.22 \\
\hline $\mathrm{T}_{9}$ & $\begin{array}{l}\text { Tricyclazole } 75 \% \text { WP } \\
(\mathrm{RC})\end{array}$ & $0.6 \mathrm{~g}$ & $\begin{array}{c}24.63 \\
(29.76)\end{array}$ & $\begin{array}{c}21.88 \\
(27.89)\end{array}$ & $\begin{array}{c}23.25 \\
(28.84)\end{array}$ & 62.46 & $\begin{array}{c}21.14 \\
(27.31)\end{array}$ & $\begin{array}{c}18.16 \\
(25.14)\end{array}$ & $\begin{array}{c}19.65 \\
(26.25)\end{array}$ & 64.24 & 3736.30 & 3856.30 & 3796.30 \\
\hline $\mathrm{T}_{10}$ & Control & - & $\begin{array}{c}62.94 \\
(52.54)\end{array}$ & $\begin{array}{c}60.94 \\
(51.36)\end{array}$ & $\begin{array}{c}61.94 \\
(51.95)\end{array}$ & - & $\begin{array}{c}55.83 \\
(48.38)\end{array}$ & $\begin{array}{c}54.08 \\
(47.37)\end{array}$ & $\begin{array}{c}54.95 \\
(47.87)\end{array}$ & - & 2597.04 & 2745.19 & 2689.63 \\
\hline & Mean & & $\begin{array}{c}31.63 \\
(33.98)\end{array}$ & $\begin{array}{c}29.99 \\
(32.91)\end{array}$ & $\begin{array}{c}30.81 \\
(33.45)\end{array}$ & & $\begin{array}{c}25.28 \\
(29.82)\end{array}$ & $\begin{array}{c}23.88 \\
(28.83)\end{array}$ & $\begin{array}{c}24.58 \\
(29.33)\end{array}$ & & 3659.84 & 3842.55 & 3773.83 \\
\hline & CV (\%) & & 5.79 & 5.75 & 5.75 & & 7.95 & 8.03 & 7.91 & & 9.74 & 9.19 & 9.12 \\
\hline & CD (0.05) & & 3.08 & 2.98 & 3.02 & & 3.52 & 3.45 & 3.45 & & 489.80 & 486.24 & 482.47 \\
\hline
\end{tabular}


Table.2 An economic analysis of combi product fungicides against blast disease of Paddy under field condition

\begin{tabular}{|c|c|c|c|c|c|c|c|c|c|c|c|c|}
\hline $\begin{array}{l}\text { Tr. } \\
\text { No. }\end{array}$ & Treatment & $\begin{array}{c}\text { Cost of } \\
\text { The } \\
\text { chemical } \\
\text { (Rs)/lt or } \\
\text { Kg }\end{array}$ & $\begin{array}{c}\text { Qty } \\
\text { required/ } \\
\text { ha* in } 2 \\
\text { spray } \\
\text { ml/gm }\end{array}$ & $\begin{array}{c}\text { Total } \\
\text { cost of } \\
\text { chemical/ } \\
\text { ha in } 2 \\
\text { spray (Rs) }\end{array}$ & $\begin{array}{c}\text { Cost of } \\
\text { cultivation } \\
\text { (Rs) }\end{array}$ & $\begin{array}{c}\text { Total } \\
\text { cost } \\
\text { (Rs.) }\end{array}$ & $\begin{array}{l}\text { Additional } \\
\text { cost over } \\
\text { control } \\
\text { (Rs.) }\end{array}$ & $\begin{array}{c}\text { Yield } \\
\text { (kg/ha) }\end{array}$ & $\begin{array}{c}\text { Total } \\
\text { returns } \\
(\mathbf{R s})^{* *}\end{array}$ & $\begin{array}{c}\text { Net } \\
\text { returns } \\
(\mathbf{R s})\end{array}$ & $\begin{array}{l}\text { Additional } \\
\text { returns } \\
\text { over } \\
\text { control } \\
\text { (Rs) } \\
\end{array}$ & B:C \\
\hline 1 & 2 & 3 & 4 & 5 & 6 & $7(5+6)$ & 8 & 9 & 10 & $11(10-7)$ & 12 & $13(10 / 7)$ \\
\hline $\mathrm{T}_{1}$ & $\begin{array}{l}\text { Flusilazole } 12.5 \% \\
+ \text { Carbendazim } \\
25 \% \text { SC }\end{array}$ & $1760 /-$ & 1000 & 1760 & 43823 & 45583 & 1760 & 3787.78 & 64392 & 18809 & 16908 & 1.41 \\
\hline $\mathrm{T}_{2}$ & $\begin{array}{l}\text { Azoxystrobin } 18.2 \\
\% \text { w/w }+ \\
\text { Difenoconazole } \\
11.4 \% \text { w/w SC }\end{array}$ & $2600 /-$ & 1000 & 2600 & 43823 & 46423 & 2600 & 4310.74 & 73283 & 26860 & 24959 & 1.58 \\
\hline $\mathrm{T}_{3}$ & $\begin{array}{l}\text { Azoxystrobin } 11 \% \\
+ \text { Tebuconazole } \\
18.3 \% \text { w/w SC }\end{array}$ & $3400 /-$ & 1500 & 5100 & 43823 & 48923 & 5100 & 4061.48 & 69045 & 20122 & 18221 & 1.41 \\
\hline $\mathrm{T}_{4}$ & $\begin{array}{l}\text { Tricyclazole } 18 \%+ \\
\text { Mancozeb } 62 \% \text { WP }\end{array}$ & $1290 /-$ & 2500 & 3225 & 43823 & 47048 & 3225 & 3777.04 & 64210 & 17162 & 15261 & 1.36 \\
\hline $\mathrm{T}_{5}$ & $\begin{array}{l}\text { Zineb } 68 \%+ \\
\text { Hexaconazole } 4 \% \\
\text { WP }\end{array}$ & $1140 /-$ & 2500 & 2850 & 43823 & 46673 & 2850 & 3477.78 & 59122 & 12449 & 10548 & 1.27 \\
\hline $\mathrm{T}_{6}$ & $\begin{array}{l}\text { Trifloxystrobin } 25 \% \\
+ \text { Tebuconazole } \\
50 \% \text { WG }\end{array}$ & $6400 /-$ & 400 & 2560 & 43823 & 46383 & 2560 & 4311.85 & 73301 & 26918 & 25017 & 1.58 \\
\hline $\mathrm{T}_{7}$ & $\begin{array}{l}\text { Mancozeb 50\% + } \\
\text { Carbendazium 25\% } \\
\text { WS }\end{array}$ & $1230 /-$ & 2500 & 3075 & 43823 & 46898 & 3075 & 3625.93 & 61641 & 14743 & 12842 & 1.31 \\
\hline $\mathrm{T}_{8}$ & $\begin{array}{l}\text { Fluxapyroxad } 62.5 \\
\text { g/l + Epoxiconazole } \\
62.5 \text { g/l EC }\end{array}$ & 1950/- & 1500 & 2925 & 43823 & 46748 & 2925 & 3922.22 & 66678 & 19930 & 18029 & 1.43 \\
\hline $\mathrm{T}_{9}$ & $\begin{array}{l}\text { Tricyclazole } 75 \% \\
\text { WP (RC) }\end{array}$ & $2400 /-$ & 600 & 1440 & 43823 & 45263 & 1440 & 3896.30 & 66237 & 20974 & 19073 & 1.46 \\
\hline $\mathrm{T}_{10}$ & Control & - & - & - & 43823 & 43823 & 0 & 2689.63 & 45724 & 1901 & 0 & 1.04 \\
\hline
\end{tabular}


Further, in the pooled data of yield observations, the highest pooled yield was recorded in Trifloxystrobin 25\% + Tebuconazole $\quad 50 \% \quad$ WG @ 0.4 $\mathrm{g} / \mathrm{L}(4311.85 \mathrm{~kg} / \mathrm{ha})$ and Azoxystrobin $18.2 \%$ w/w +Difenoconazole 11.4\% w/w SC @ 1.0 $\mathrm{g} / \mathrm{L}(4310.74 \mathrm{~kg} / \mathrm{ha})$ followed by Tricyclazole 75\% WP @ 0.6 g/L(3796.30 kg/ha) when compared to control (2689.63 kg/ha). The least pooled grain yield was observed in Zineb 68\% + Hexaconazole 4\% WP@ 2.5 g/L $(3477.78 \mathrm{~kg} / \mathrm{ha})$ when compared to other treatments (Table 1 and Plate 1).

All the treatments investigated under field condition showed significant differences in blast disease reduction and grain yield. The results obtained are also in agreement with the work of $(14,15)$ who also reported the complete inhibition of growth of Pyricularia oryzae in Trifloxystrobin $25 \%+$ Tebuconazole $50 \% \mathrm{WG}$ and Tricyclazole $75 \%$ WPas effective fungicides against Pyricularia oryzae.

\section{Economics of fungicidal evaluation}

The economics of cost benefit ratio has been worked out for different fungicides and are presented in Table 2. The highest total returns were obtained by Trifloxystrobin $25 \%+$ Tebuconazole 50\% $\quad$ WG @ 0.4 g/L(Rs. 73,301) followed byAzoxystrobin $18.2 \%$ w/w +Difenoconazole 11.4\% w/w SC @ 1.0 g/L(Rs.73,283). Similarly net returns and additional net returns over control were also high in Trifloxystrobin $25 \%+$ Tebuconazole $50 \%$ WG @ 0.4 g/L (Rs. 26,918 and Rs. 25,017 respectively) followed by Azoxystrobin $18.2 \%$ w/w +Difenoconazole 11.4\% w/w SC @ 1.0 g/L (Rs. 26,860 and Rs. 24,959 respectively) than any other fungicides. However, when cost benefit ratio was calculated, Trifloxystrobin 25\% + Tebuconazole $50 \% \quad \mathrm{WG} \quad$ (1.58) and Azoxystrobin $18.2 \% \mathrm{w} / \mathrm{w}+$ Difenoconazole
11.4\% w/w SC @ 1.0 g/L(1.58) proved better because of curative effect, combi product and systemic in nature of the chemical than any other fungicides.

However from the farmer's point of view, the economics of disease management is important. In the present investigation the Trifloxystrobin 25\% + Tebuconazole 50\% WG @ 0.4 g/L has given highest total returns, net returns and additional returns over control than any other fungicides. The Azoxystrobin $18.2 \% \mathrm{w} / \mathrm{w}+$ Difenoconazole $11.4 \% \mathrm{w} / \mathrm{w}$ SC @ $1.0 \mathrm{~g} / \mathrm{Lw}$ as next in order with respect to all the three above mentioned parameters. This is obviously due to their mode of action and also lowering of both leaf and neck blast incidence.

\section{References}

1. Abodolereza, A. and Racionzer, P., 2009, Food outlook: Global market analysis. pp. 23-27.

2. Anonymous. Statistical database. 2017; http://www.fao.org.

3. Hollier CA, Groth DE, Rush MC, Webster AK. Common Names of Plant Diseases. The American Phytopathological Society, St. Paul, MN, 1984.

4. Gilbert MJ, Soanes DM, Talbot NJ. Functional Genomic Analysis of the Rice Blast Fungus Magnaporthegrisea. Appl. Mycol. and Biotechnol.2004; 4: 331-352.

5. Padmanabhan SY. Estimating losses from rice blast in India. Pages 203-221 in the rice blast disease. Proceeding of symposium at IRRI, 1965.

6. Ou SH. Rice Diseases, CAB International Mycological, Institute Kew, Survey, UK. Johan Hopkins Press, Baltinoie, Maryland. 1985, 203-221.

7. Awodera VA, Esuruoso OF. Reduction in grain yield of two rice varieties infected by rice blast disease in Nigeria. 
Nigerian Agric J. 1975; 11:170-173.

8. Samira S, Afifa M, Amina D, Allal D. In vivo effect of fungicides on the development of Pyricularia grisea and Heliminthosporiumoryzae.Phytopathol. Mediterranian.2002; 41:235-246.

9. Bonman JM, Estrada BA, Banding JM. Leaf and neck blast resistance in tropical lowland rice cultivars. Plant Dis. 1989; 73:388-390.

10. Georgopoulos SG, Ziogas BN. Principles and methods for control of plant diseases, Athens. 1992, 236.

11. Naidu VD, Reddy GV. Control of blast (BI) in main field and nursery with some new fungicides. R.P.P. 1989; 69: 209.

12. Wheeler BEJ. An Introduction to Plant
Diseases. John Wiley and Sons, Ltd. London. 1969, 301.

13. Panse VG, Sukathme PV. Statistical methods for agricultural workers. ICAR, New Delhi. 1967, 145-155.

14. Neelakanth, Sidde Gowda DK, Chethana $\mathrm{BS}$, Parasappa $\mathrm{HH}$. In vitro and in vivo evaluation of fungicides against pyricularia oryzae causing blast of rice. Int. J. Pure App. Biosci. 2017; 5(3):259-263.

15.Wasimfiroz RM, Hosagoudar GN, Narayanaswamy $\mathrm{H}$ and Balanagouda Patil. Evaluation of fungicides, bioagents and plant extracts against Pyricularia oryzae. International Journal of Chemical Studies, 2018; 6(2): 1875-1879.

\section{How to cite this article:}

Hosagoudar, G.N. 2019. Investigation of Combi Product Fungicides for the Management of Blast Diseases of Paddy. Int.J.Curr.Microbiol.App.Sci. 8(07): 2128-2134.

doi: https://doi.org/10.20546/ijcmas.2019.807.256 